\title{
ARITHMETICAL STUDY \\ OF A CERTAIN TERNARY RECURRENCE SEQUENCE AND RELATED QUESTIONS
}

\author{
M. MIGNOTTE AND N. TZANAKIS
}

\begin{abstract}
The complete solution in $\left(n, y_{1}, y_{2}\right) \in \mathbf{Z}^{3}$ of the Diophantine equation

$$
b_{n}= \pm 2^{y_{1}} 3^{y_{2}}
$$
\end{abstract}

is given, where $\left(b_{n}\right)_{n \in \mathbf{Z}}$ is Berstel's recurrence sequence defined by

$$
b_{0}=b_{1}=0, \quad b_{2}=1, \quad b_{n+3}=2 b_{n+2}-4 b_{n+1}+4 b_{n} .
$$

\section{INTRODUCTION}

Let $\left(u_{n}\right)_{n \in \mathbf{Z}}$ be a linear recurrence sequence in $\mathbf{Q}$ whose characteristic polynomial has at least two distinct roots and suppose that this sequence is nondegenerate, i.e., the ratio of any distinct roots of the characteristic polynomial is not a root of unity. Let $c$ be an integer which is either a constant or an $S$-integer (i.e., an integer whose prime divisors belong to a finite fixed set of primes). Under these assumptions, the equation $u_{n}=c$ (in the unknown $n$ ) has at most finitely many solutions; see, for example, Corollary 3 of J. H. Evertse [3]. The problem of the explicit computation of these solutions is a difficult one, and in a previous paper of ours [6] we propose a general practical method for the explicit solution of equations as above. The purpose of our present paper is to give an interesting application of our method [6] to the equation

$$
b_{n}= \pm 2^{r} 3^{s},
$$

where $\left(b_{n}\right)_{n \in \mathbf{Z}}$ is Berstel's ternary recurrence sequence defined by

$$
b_{0}=b_{1}=0, \quad b_{2}=1, \quad b_{n+3}=2 b_{n+2}-4 b_{n+1}+4 b_{n} .
$$

We quote from the introduction of our paper [6]: “Among ternary linear recurrence sequences, it seems that Berstel's sequence...plays a very special role. Firstly, it is the only known example of a nondegenerate ternary linear recurrence sequence which has six zeros (by definition, a nondegenerate linear recurrence sequence has only finitely many zeros). It was proved in [4] that it contains exactly six zeros. F. Beukers has just proved [in the meantime, this has appeared in [1]] that six is the right upper bound for the number of zeros of nondegenerate ternary recurrence sequences of integers. Secondly, Berstel's sequence contains

Received by the editor February 18, 1992.

1991 Mathematics Subject Classification. Primary 11B37, 11 D61.

(c) 1993 American Mathematical Society $0025-5718 / 93 \$ 1.00+\$ .25$ per page 
many repetitions; indeed it was proved in [5] that the equation $b_{m}= \pm b_{n}$ for rational integers $m, n \in \mathbf{Z}$ has exactly 21 solutions $(m, n)$ with $m<n$, and these solutions were explicitly computed. For the problem studied here, i.e., the equation $u_{n}= \pm 2^{r} 3^{s}$, it seems again that Berstel's sequence has remarkable properties: we can prove that there are exactly 44 solutions $(n, r, s)$." In our aforementioned paper we announce without proof the complete solution of (1) (see the theorem in §IV of [6]). Here we will give all the details of the solution. In particular, we hope to make clear, by means of the concrete example which we study, the part of our method described only in general terms in the remark of §III of [6].

\section{Preliminaries}

We work in the field $Q(\theta)$, where $\theta^{3}-2 \theta^{2}+4 \theta-4=0$. In this field, $\pi=\theta^{2} / 2$ is a prime element and $(2)=\pi^{3}$. More precisely, $2=\pi^{3} \varepsilon$, where $\varepsilon=3-\theta+\theta^{2}$ is a unit and $\theta=\pi^{2} \mu$, where $\mu=1+\theta^{2} / 2$ is a unit $\left(1, \theta, \theta^{2} / 2\right.$ is an integral basis in the field $Q(\theta))$. If $\theta^{(1)}, \theta^{(2)}, \theta^{(3)}$ are the conjugates of $\theta$ in $\mathbf{C}$ (exactly one is real), then it is easy to see that

$$
b_{n}=\sum_{i=1}^{3} \alpha_{i} \theta^{(i)^{n}}, \quad \text { where } \alpha_{1}=\frac{\theta^{(3)}-\theta^{(2)}}{4 \sqrt{-11}}, \alpha_{2}=\frac{\theta^{(3)}-\theta^{(1)}}{4 \sqrt{-11}}, \alpha_{3}=\frac{\theta^{(2)}-\theta^{(1)}}{4 \sqrt{-11}} \text {. }
$$

We write $n=3 m+j$, with $j \in\{0,1,2\}$. Then

$$
\begin{aligned}
b_{n} & =\sum_{i=1}^{3} \alpha_{i} \pi^{(i)^{6 m+2 j}} \mu^{(i)^{3 m+j}}=\sum_{i=1}^{3} \alpha_{i}\left(\pi^{(i)^{2}} \mu^{(i)}\right)^{j}\left(\pi^{(i)^{3}}\right)^{2 m} \mu^{(i)^{3 m}} \\
& =\sum_{i=1}^{3} \alpha_{i}\left(\pi^{(i)^{2}} \mu^{(i)}\right)^{j}\left(2 \varepsilon^{(i)^{-1}}\right)^{2 m} \mu^{(i)^{3 m}} \\
& =2^{2 m} \sum_{i=1}^{3} \alpha_{i}\left(\pi^{(i)^{2}} \mu^{(i)}\right)^{j}\left(\mu^{(i)^{3}} \varepsilon^{(i)^{-2}}\right)^{m}=2^{2 m} \sum_{i=1}^{3} \alpha_{i} \theta^{(i)^{j}} \omega^{(i)^{m}},
\end{aligned}
$$

where $\omega=\mu^{3} \varepsilon^{-2}$ and $\omega^{3}+\omega^{2}+\omega-1=0$.

Thus, for $j=1,2, b_{3 m+j}=2^{2 m} u_{j m}$, where $u_{j m}$ is given by the formula

$$
u_{j m}=\sum_{i=1}^{3} \alpha_{i} \theta^{(i)^{j}} \omega^{(i)^{m}}
$$

and

$$
b_{3 m}=2^{2 m-1} u_{0 m}, \quad \text { where } u_{0 m}=\sum_{i=1}^{3} 2 \alpha_{i} \omega^{(i)^{m}},
$$

and in all the three cases, $u_{j, m+3}=-u_{j, m+2}-u_{j, m+1}+u_{j, m}$.

It is easy to see that $\left(u_{j 0}, u_{j 1}, u_{j 2}\right)=(0,1,0),(0,0,1),(1,-1,1)$ according as $j=0,1,2$, respectively. In the following sections we shall solve the equations

$$
u_{j m}= \pm 2^{r} 3^{s}
$$

for each value $j \in\{0,1,2\}$ separately. The advantage of working with the sequence $\left(u_{j m}\right)$ instead of $\left(b_{n}\right)$ is that the first one assumes only integral values, 
even for negative index $n$. For simplicity in our notations we will omit the index $j$, but in the beginning of each section it will be clear which sequence we study.

In the present paper we will often apply Theorem 1 of $\S$ II of [6]. We describe its use in our situation: Let $p$ be a prime $\neq 2,11$ (these are the primes dividing the discriminant of the minimal polynomial of $\omega$ ). Choose a positive integer $S$ such that $\omega^{S} \equiv A(\bmod p)$ for some $A \in \mathbf{Z}$. Suppose, moreover, that $A$ has been chosen in such a way that the orders of $A$ modulo $p$ and $p^{2}$ have the same value $R$. Then we have the following result (cf. Theorem 1, §II of [6]):

Theorem 1. Let the rational integer $c$ be such that either $c \not \equiv 0(\bmod p)$ or $c=0$. Let $\mathscr{P}$ be a complete system of residues modulo $S$, and $\mathscr{M}$ a subset of $\mathscr{P}$ satisfying the following conditions:

(i) $u_{m}=c$ for every $m \in \mathscr{M}$,

(ii) if $n \in \mathscr{P}$ and $u_{n} \equiv c A^{r}(\bmod p)$ for some $r \in\{0,1, \ldots, R-1\}$, then $n \in \mathscr{M}$,

(iii) $u_{m+S} \not \equiv A u_{m}\left(\bmod p^{2}\right)$ for every $m \in \mathscr{M}$.

Then $u_{n}=c$ implies $n \in \mathscr{M}$.

In the beginning of $\S 4$ we will use another result from [6] (Theorem 2 of [6]):

Theorem 2. Let $p, \omega$, and $A$ be as in Theorem 1 and $\mathscr{N}=\left\{n \in \mathbf{Z}: u_{n}=0\right\}$. Let $q$ be a prime $\neq p$ and $\nu$ a positive integer such that the following condition is satisfied:

$$
u_{m} \equiv 0\left(\bmod q^{\nu}\right) \Rightarrow \exists n \in \mathscr{N} \text { such that } n \equiv m(\bmod S) .
$$

Then $u_{m} \equiv 0\left(\bmod q^{\nu}\right)$ implies that $p$ divides $u_{m}$.

Remark. More often in this paper we will use, instead of Theorem 2, the following trick (cf. with the remark of $\S$ III of [6]): Let $p$ be a prime. Then $\left(u_{n}\right)$ is periodic modulo $p$, with period $P$, say. Next, consider a prime $q \neq p$. The sequence $\left(u_{n}\right)$ is periodic modulo $q^{\nu}$ for any positive integer $\nu$, with period length $Q$, say (depending on $\nu$ ) ; therefore, a relation of the form $u_{n} \equiv 0$ $\left(\bmod q^{\nu}\right)$ restricts the values of the index $n$ modulo $Q$, hence, if $\operatorname{gcd}(P, Q)$ is not "very small", restricts the values of $n$ modulo $P$ to only "a few" possibilities, say $n_{1}, \ldots, n_{k}(\bmod P)$. With a convenient choice of the prime $p$, it can happen that $p$ divides $u_{n_{j}}$ for every $j=1, \ldots, k$, and in this case we get the same conclusion as in Theorem 2 , i.e.,

$$
u_{n} \equiv 0\left(\bmod q^{\nu}\right) \Rightarrow u_{n} \equiv 0(\bmod p) .
$$

Thus, if it is known a priori that $u_{n}$ is not divisible by $p$, we conclude that a power of $q$ can divide $u_{n}$ only if it is lower than $q^{\nu}$.

\section{The CASE $j=0$}

Here we have

$$
u_{0}=0, \quad u_{1}=1, \quad u_{2}=0, \quad u_{m+3}=-u_{m+2}-u_{m+1}+u_{m} .
$$

We apply first the remark at the end of $\S 2$, with $q=2, \nu=3, Q=16$, $p=7$, and $P=48$ to conclude that if 8 divides $u_{m}$, then 7 also divides $u_{m}$. Therefore, we can assume $0 \leq r \leq 2$. 
To find an upper bound for $s$ using Theorem 2 or the above remark seems difficult in this case. Therefore, we work as follows, distinguishing three cases. First note that for $s \geq 2$ one has necessarily $m \equiv 0,2(\bmod 13)$.

(i) $m \equiv 0(\bmod 13)$ and $s \geq 7$. In this case,

$$
u_{m} \equiv 0\left(\bmod 3^{7}\right) \Rightarrow m \equiv 0(\bmod 13 \cdot 81) \Rightarrow m \equiv 0,81(\bmod 162) \text {. }
$$

Since $m \equiv 0(\bmod 162)$ implies that 163 divides $u_{m}$, we have $m \equiv 81(\bmod$ 162).

(ii) $m \equiv 2(\bmod 13)$ and $s \geq 6$. In this case an argument similar to the previous one shows that we must have $m \equiv 83(\bmod 162)$.

(iii) $0 \leq s \leq 6$. This case can be treated as $\S I I$ of [6] suggests (see below).

First we exclude the first two cases. To simplify notations, we shall write

$$
(a, b) \equiv\left(a^{\prime}, b^{\prime}\right) \quad \bmod \left(m_{1}, m_{2}\right)
$$

instead of the two relations

$$
a \equiv a^{\prime}\left(\bmod m_{1}\right) \& b \equiv b^{\prime}\left(\bmod m_{2}\right) .
$$

Note that in both cases (i) and (ii) the index $m$ is odd, which implies that $u_{m}$ is also odd, and therefore $r=0$. Moreover, we have

In case (i),

$$
\begin{aligned}
m \equiv 81(\bmod 162) & \Rightarrow u_{m} \equiv 15(\bmod 163) \\
& \Rightarrow 3^{s} \equiv \pm 15(\bmod 163) \Rightarrow s \equiv 22(\bmod 81)
\end{aligned}
$$

therefore

$$
(m, s) \equiv(9,4) \quad \bmod (18,9)
$$

We have the table

\begin{tabular}{|c|r|r|r|r|r|r|r|r|r|r|r|r|}
\hline$m$ & 9 & 27 & 45 & 63 & 81 & 99 & 117 & 135 & 153 & 171 & 189 & $\bmod 198$ \\
\hline$u_{m}$ & -3 & 21 & 97 & -80 & -41 & 91 & 10 & -92 & 79 & -8 & -74 & $\bmod 199$ \\
\hline$s$ & 100 & 143 & 131 & 67 & 68 & 116 & 46 & 33 & 61 & 21 & 18 & $\bmod 199$ \\
\hline
\end{tabular}

The only pair $(m, s)$ in this table which satisfies $(4)$ is $(63,67) \bmod (198,99)$.

On the other hand, $m \equiv 63(\bmod 198)$ implies $m \equiv 63,129(\bmod 132)$ and, making use of the auxiliary prime $397=3 \cdot 132+1$, we see that

$$
\begin{aligned}
m \equiv 63(\bmod 132) & \Rightarrow u_{m} \equiv-156(\bmod 397) \Rightarrow 3^{s} \equiv \pm 156(\bmod 397) \\
& \Rightarrow s \cdot \operatorname{ind} 3=\operatorname{ind}( \pm 156)(\bmod 396) \Rightarrow s \equiv 2(\bmod 9),
\end{aligned}
$$

which contradicts $s \equiv 67(\bmod 99)$.

If $m \equiv 129(\bmod 132)$, we get analogously $s \equiv 1(\bmod 9)$, which is again a contradiction.

In case (ii) we work as in case (i) to obtain first

$$
(m, s) \equiv(11,8) \bmod (18,9) \text {, }
$$

and then we construct a table relative to the auxiliary prime 199 , from which we see that the only pair which satisfies (5) is

$$
(m, s) \equiv(155,80) \quad \bmod (198,99) \text {. }
$$


In particular, $m \equiv 23,89(\bmod 132)$ and, as before, we make use of the auxiliary prime 397 . If $m \equiv 23(\bmod 132)$, we easily get $s \equiv 0(\bmod 9)$, which contradicts $(6)$, and if $m \equiv 89(\bmod 132)$, then $s \equiv 10(\bmod 11)$, which again contradicts (6) (note that 396 is divisible by 99 ).

Now that we have excluded cases (i) and (ii), we are left with (iii); i.e., we have to solve

$$
u_{m}= \pm 2^{r} \cdot 3^{s}, \quad 0 \leq r \leq 2,0 \leq s \leq 6 .
$$

We applied Theorem 1 of [6] with $p \in\{47,53,103,163,199,397\}$, using a simple computer program. The values of the various parameters and a summary of the application of that theorem to the solution of (7) are given, respectively, in Tables I and II.

TABLE I

\begin{tabular}{|c|c|c|c|}
\hline$p$ & $S$ & $A$ & $R$ \\
\hline 47 & 46 & 1 & 1 \\
\hline 53 & 52 & 1 & 1 \\
\hline 103 & 17 & 56 & 3 \\
\hline 163 & 54 & -59 & 3 \\
\hline 199 & 66 & -93 & 3 \\
\hline 397 & 132 & 1 & 1 \\
\hline
\end{tabular}

TABLE II. $u_{m}= \pm 2^{r} 3^{s}, 0 \leq r \leq 2,0 \leq s \leq 6$

\begin{tabular}{|r|r||c|c||r|r|}
\hline \multicolumn{2}{|c||}{$u_{m}$} & \multicolumn{2}{c||}{$m$} & \multicolumn{2}{r|}{$p$} \\
\hline 1 & -1 & $1,-1$ & 3,5 & 53 & 103 \\
\hline 2 & -2 & $4,-2$ & 6,12 & 53 & 53 \\
\hline 3 & -3 & -3 & 9 & 199 & 103 \\
\hline 4 & -4 & $\varnothing$ & 8 & 47 & 53 \\
\hline 6 & -6 & -4 & $\varnothing$ & 53 & 47 \\
\hline 9 & -9 & 15 & $\varnothing$ & 163 & 199 \\
\hline 12 & -12 & 10 & $\varnothing$ & 53 & 47 \\
\hline 18 & -18 & $\varnothing$ & $\varnothing$ & 47 & 47 \\
\hline 27 & -27 & 13 & $\varnothing$ & 103 & 53 \\
\hline 36 & -36 & $\varnothing$ & $\varnothing$ & 47 & 53 \\
\hline 54 & -54 & $\varnothing$ & $\varnothing$ & 47 & 47 \\
\hline 81 & -81 & $\varnothing$ & $\varnothing$ & 53 & 47 \\
\hline 108 & -108 & $\varnothing$ & $\varnothing$ & 47 & 47 \\
\hline 162 & -162 & $\varnothing$ & $\varnothing$ & 103 & 103 \\
\hline 243 & -243 & $\varnothing$ & $\varnothing$ & 47 & 47 \\
\hline 324 & -324 & $\varnothing$ & $\varnothing$ & 103 & 53 \\
\hline 486 & -486 & $\varnothing$ & $\varnothing$ & 47 & 103 \\
\hline 729 & -729 & $\varnothing$ & $\varnothing$ & 47 & 47 \\
\hline 972 & -972 & $\varnothing$ & $\varnothing$ & 47 & 47 \\
\hline 1458 & -1458 & $\varnothing$ & $\varnothing$ & 103 & 53 \\
\hline 2916 & -2916 & $\varnothing$ & $\varnothing$ & 103 & 103 \\
\hline
\end{tabular}


The symbol $\varnothing$ in the second main column means that the congruence $u_{m} \equiv c$ $(\bmod p)$, where $c$ and $p$ are the numbers of the first and third main column on the same row, respectively, is impossible.

\section{The CASES $j=1$ AND $j=2$}

Here we have

$$
\begin{aligned}
& j=1: \quad u_{0}=0, \quad u_{1}=0, \quad u_{2}=1, \quad u_{m+3}=-u_{m+2}-u_{m+1}+u_{m}, \\
& j=2: \quad u_{0}=1, \quad u_{1}=-1, \quad u_{2}=1, \quad u_{m+3}=-u_{m+2}-u_{m+1}+u_{m} .
\end{aligned}
$$

This section is mainly devoted to the case $j=1$. The case $j=2$ is very easy, and its solution is given at the end of the section.

The case $j=1$.

First we solve the equation

$$
u_{m}= \pm 3^{s}
$$

It is easily checked that

$$
u_{m} \equiv 0\left(\bmod 3^{8}\right) \Rightarrow m \equiv 0,1,4,17\left(\bmod 3^{4} \cdot 13\right) .
$$

In particular, if $s \geq 8$, either $m \equiv 0,1,4,17$ or $m \equiv 81,82,85,98$ $(\bmod 162)$. In the first case, the hypotheses of Theorem 2 of [6] are satisfied with $q=3, p=163, S=162, A=1$, and $\nu=8$, and we conclude that 163 divides $u_{m}$, which contradicts (8). Thus, we are left with the following cases:

(i) $s \geq 8$ and $m \equiv 81,82,85,98(\bmod 162)$,

(ii) $s \leq 7$.

We show that (i) is impossible, as we did in (i) and (ii) when $j=0$. We have,

$$
\begin{aligned}
m \equiv 81(\bmod 162) & \Rightarrow u_{m} \equiv-61(\bmod 163) \\
& \Rightarrow 3^{s} \equiv \pm 61(\bmod 163) \Rightarrow s \equiv 12(\bmod 81) .
\end{aligned}
$$

Analogously,

$$
\begin{aligned}
& m \equiv 82(\bmod 162) \Rightarrow s \equiv 59(\bmod 81), \\
& m \equiv 85(\bmod 162) \Rightarrow s \equiv 38(\bmod 81), \\
& m \equiv 98(\bmod 162) \Rightarrow s \equiv 1(\bmod 81) .
\end{aligned}
$$

Therefore, only the following cases are possible:

$$
(m, s) \equiv(9,3),(10,5),(13,2),(8,1) \bmod (18,9) .
$$

Then, we work modulo 199 , exactly as we did immediately after (4), to conclude that the fourth case above is impossible and, corresponding to the first three cases, we have respectively

$$
\begin{aligned}
& (m, s) \equiv(9,21),(171,21) \bmod (198,99), \\
& (m, s) \equiv(190,5) \bmod (198,99), \\
& (m, s) \equiv(157,92) \bmod (198,99) .
\end{aligned}
$$

In case (9) we have $m \equiv 9,171,207,369(\bmod 396)$, which implies

$$
m \equiv 9,39,75,105 \quad(\bmod 132) \text {. }
$$


Analogously, in cases (10) and (11) we have, respectively,

$$
\begin{aligned}
& m \equiv 58,124 \quad(\bmod 132), \\
& m \equiv 25,91 \quad(\bmod 132) .
\end{aligned}
$$

Then we work modulo 397 to show that each pair of the relations (9) \& (12), (10) \& (13), and (11) \& (14), is either contradictory or includes an impossible relation. We give some typical examples (note that the order of 3 modulo 397 is 198 and that $-1 \equiv 3^{99}(\bmod 397)$ :

$$
m \equiv 9(\bmod 132) \Rightarrow u_{m} \equiv-8(\bmod 397) \Rightarrow 3^{s} \equiv \pm 8(\bmod 397)
$$

and the last relation is impossible;

$$
\begin{aligned}
m \equiv 75(\bmod 132) & \Rightarrow u_{m} \equiv-136(\bmod 397) \Rightarrow 3^{s} \equiv \pm 136(\bmod 397) \\
& \Rightarrow s \equiv 114(\bmod 198) \Rightarrow s \equiv 6(\bmod 9),
\end{aligned}
$$

while (9) implies that $s \equiv 3(\bmod 9)$;

$$
\begin{aligned}
m \equiv 91(\bmod 132) & \Rightarrow u_{m} \equiv 179(\bmod 397) \Rightarrow 3^{s} \equiv \pm 179(\bmod 397) \\
& \Rightarrow s \equiv 47(\bmod 198) \Rightarrow s \equiv 3(\bmod 11),
\end{aligned}
$$

while from $(11)$ we must have $s \equiv 4(\bmod 11)$. All the remaining cases are treated analogously.

Now we are left with case (ii) of (8). We deal with it as we did with equation (7), and we summarize its solution in Table III.

The equation

$$
u_{m}= \pm 2^{r}
$$

is easier to solve than (8). First we observe that

$$
u_{m} \equiv 0\left(\bmod 2^{4}\right) \Rightarrow u_{m} \equiv 0(\bmod 7) \text {, }
$$

which implies that $r \leq 3$ in (15), and this equation's solution is summarized in Table IV.

TABLE III. $u_{m}= \pm 3^{s}, 0 \leq s \leq 7$

\begin{tabular}{|r|r||c|r||r|r|}
\hline \multicolumn{2}{|c||}{$u_{m}$} & \multicolumn{2}{c||}{$m$} & \multicolumn{2}{|c|}{$p$} \\
\hline 1 & -1 & $-2,-1,2,7$ & 3 & 47 & 47 \\
\hline 3 & -3 & $\varnothing$ & 6 & 47 & 397 \\
\hline 9 & -9 & 14 & $\varnothing$ & 163 & 103 \\
\hline 27 & -27 & $\varnothing$ & $\varnothing$ & 103 & 103 \\
\hline 81 & -81 & $30,-9$ & $\varnothing$ & 53 & 53 \\
\hline 243 & -243 & $\varnothing$ & $\varnothing$ & 53 & 53 \\
\hline 729 & -729 & $\varnothing$ & $\varnothing$ & 53 & 47 \\
\hline 2187 & -2187 & $\varnothing$ & $\varnothing$ & 47 & 47 \\
\hline
\end{tabular}

TABLE IV. $u_{m}= \pm 2^{r}, 1 \leq r \leq 3$

\begin{tabular}{|l|l||c|c||c|r|}
\hline \multicolumn{2}{|c||}{$u_{m}$} & \multicolumn{2}{c||}{$m$} & \multicolumn{2}{c|}{$p$} \\
\hline 2 & -2 & $5,-3$ & $\varnothing$ & 53 & 53 \\
\hline 4 & -4 & $8,-4$ & $\varnothing$ & 53 & 53 \\
\hline 8 & -8 & $\varnothing$ & 9 & 53 & 163 \\
\hline
\end{tabular}


Finally, we are left with the equation

$$
u_{m}= \pm 2^{r} \cdot 3^{s}, \quad 1 \leq r \leq 3, s \geq 1 .
$$

A main difficulty lies in the problem of finding an upper bound for the exponent $s$. In $\S 6$, we discuss an alternative approach to the solution of (17). We observe the following fact:

$$
\begin{aligned}
u_{m} \equiv 0(\bmod 9) & \Rightarrow m \equiv 0,1,4(\bmod 13) \\
& \Rightarrow m \equiv 0,1,4,13,14,17,26,27,30,39,40,43(\bmod 52) .
\end{aligned}
$$

The values $m \equiv 0,1,4,17(\bmod 52)$ are rejected because, for such values, 53 divides $u_{m}$. On the other hand, from (17), $u_{m}$ is even, which implies that $m \equiv 0,1(\bmod 4)$. Thus, we are left with

$$
m \equiv 13,40 \quad(\bmod 52) \text {. }
$$

Also, if $3^{5}$ divides $u_{m}$, then $m \equiv 0,1,4,17(\bmod 39)$ and the values $m \equiv$ $4,17(\bmod 39)$ are rejected in view of $(18)$. Finally, if $3^{6}$ divides $u_{m}$ and $m \equiv 0,1(\bmod 39)$, then $m \equiv 0,1\left(\bmod 13 \cdot 3^{4}\right)$. Therefore, in order to solve (16), we distinguish two cases:

(i) $s \geq 6$, and consequently $m \equiv 0,1(\bmod 13 \cdot 81)$,

(ii) $s \leq 5$.

First we exclude case (i), working modulo 163,199 , and 397, successively. Notice that in case (i) we have, in particular, $m \equiv 81,82(\bmod 162)$, as we noticed in the resolution of equation (8).

The number 3 is a primitive root for the modulus 163; therefore,

$$
\begin{aligned}
m \equiv 81(\bmod 162) & \Rightarrow u_{m} \equiv-61(\bmod 163) \Rightarrow 2^{r} 3^{s} \equiv \pm 61(\bmod 163) \\
& \Rightarrow 3^{77 r+s} \equiv \pm 61(\bmod 163) \Rightarrow 77 r+s \equiv 12(\bmod 81) .
\end{aligned}
$$

From this last congruence we see that

$$
s \equiv 7,2,6(\bmod 9) \text { according as } r=1,2,3 \text {, respectively. }
$$

Analogously, we find that if $m \equiv 82(\bmod 162)$, then

$$
s \equiv 0,4,8(\bmod 9) \text { according as } r=1,2,3 \text {, respectively. }
$$

Thus, we have the following three cases:

$$
\begin{aligned}
& r=1 \quad \text { and }(m, s) \equiv(9,7),(10,0) \bmod (18,9), \\
& r=2 \text { and }(m, s) \equiv(9,2),(10,4) \bmod (18,9), \\
& r=3 \text { and }(m, s) \equiv(9,6),(10,8) \bmod (18,9) .
\end{aligned}
$$

Next we work modulo 199. Equation (17) implies $u_{m} \equiv \pm 3^{7 r+s}(\bmod 199)$. We have the following table when $m \equiv 9(\bmod 18)$ :

\begin{tabular}{|r|r|r|r|r|r|r|r|r|r|r|r|r|}
\hline$m$ & 9 & 27 & 45 & 63 & 81 & 99 & 117 & 135 & 153 & 171 & 189 & $\bmod 198$ \\
\hline$u_{m}$ & -8 & -43 & 63 & 13 & -7 & 86 & -38 & -92 & -47 & -8 & 81 & $\bmod 199$ \\
\hline $7 r+s$ & 21 & 88 & 45 & 73 & 43 & 85 & 62 & 33 & 76 & 21 & 4 & $\bmod 99$ \\
\hline $7 r+s$ & 3 & 7 & 0 & 1 & 7 & 4 & 8 & 6 & 4 & 3 & 4 & $\bmod 9$ \\
\hline
\end{tabular}


When $m \equiv 10(\bmod 18)$, we have the table

\begin{tabular}{|r|r|r|r|r|r|r|r|r|r|r|r|r|}
\hline$m$ & 10 & 28 & 46 & 64 & 82 & 100 & 118 & 136 & 154 & 172 & 190 & $\bmod 198$ \\
\hline$u_{m}$ & 5 & 64 & 34 & -93 & -34 & 5 & 48 & 0 & -73 & 0 & 44 & $\bmod 199$ \\
\hline $7 r+s$ & 39 & 42 & 31 & 66 & 31 & 39 & 29 & $*$ & 52 & $*$ & 5 & $\bmod 99$ \\
\hline $7 r+s$ & 3 & 6 & 4 & 3 & 4 & 3 & 2 & $*$ & 7 & $*$ & 5 & $\bmod 9$ \\
\hline
\end{tabular}

(an asterisque means that, modulo 199, the value $u_{m}$ is not a power of 3). From the above tables it is easy to check the following facts:

$$
\begin{aligned}
& \text { relation }(19) \text { is possible only if } m \equiv 154(\bmod 198), \\
& \text { relation }(20) \text { is possible only if } m \equiv 27,81(\bmod 198), \\
& \text { relation }(21) \text { is possible only if } m \equiv 45,118(\bmod 198) \text {. }
\end{aligned}
$$

The three relations (22), (23), and (24) imply respectively

$$
m \equiv 22,88 ; 27,93,15,81 ; 45,111,52,118(\bmod 132) .
$$

Since $u_{m}$ is even (by $\left.(17)\right)$, we must have $m \equiv 0,1(\bmod 4)$, and consequently the following cases are left:

$$
\begin{aligned}
& r=1 \quad \text { and }(m, s) \equiv(88,0) \bmod (132,9), \\
& r=2 \text { and }(m, s) \equiv(93,2),(81,2) \bmod (132,9), \\
& r=3 \text { and }(m, s) \equiv(45,6),(52,8) \bmod (132,9) .
\end{aligned}
$$

Finally we work modulo 397 . Relation (17) implies

$$
t \cdot \operatorname{ind}(-1)+r \cdot \operatorname{ind}(2)+s \cdot \operatorname{ind}(3) \equiv \operatorname{ind}\left(u_{m}\right) \quad(\bmod 396),
$$

where $t \in\{0,1\}$. Since $\operatorname{ind}(-1) \equiv \operatorname{ind}(2) \equiv 0(\bmod 9)$ and $\operatorname{ind}(3) \equiv 2$ $(\bmod 9)$, we must have

$$
2 s \equiv \operatorname{ind}\left(u_{m}\right) \quad(\bmod 9) .
$$

If $(25)$ is true, then we have the following implications:

$$
\begin{aligned}
m \equiv 88(\bmod 132) & \Rightarrow u_{m} \equiv 33(\bmod 397) \Rightarrow \operatorname{ind}\left(u_{m}\right) \equiv 322(\bmod 396) \\
& \Rightarrow \operatorname{ind}\left(u_{m}\right) \equiv 7(\bmod 9) \Rightarrow s \equiv 8(\bmod 9)
\end{aligned}
$$

(in view also of (28)), and this contradicts (25). In an analogous way we prove the impossibility of (26) and (27), and this shows that case (i) (i.e., $s \geq 6$ ) is impossible.

It remains therefore to solve the equation $u_{m}= \pm 2^{r} 3^{s}$ with $1 \leq r \leq 3$ and $1 \leq s \leq 5$. The usual table corresponding to this equation is found in Table V.

The case $j=2$. This is the easiest case. It is obvious that $u_{m}$ is odd for every $m$; therefore, we have to solve the equation $u_{m}= \pm 3^{s}$. It is easily checked that $u_{m}$ is never divisible by 27 . Therefore, $0 \leq s \leq 2$. The usual table of solutions is given in Table VI. 
TABLE V. $(j=1) u_{m}= \pm 2^{r} 3^{s}, 1 \leq r \leq 3,0 \leq s \leq 3$

\begin{tabular}{|r|r|||r|r||r|r|}
\hline \multicolumn{3}{|c||}{$u_{m}$} & \multicolumn{2}{c||}{$m$} & \multicolumn{2}{r|}{$p$} \\
\hline 6 & -6 & $\varnothing$ & $\varnothing$ & 47 & 47 \\
\hline 12 & -12 & $\varnothing$ & $\varnothing$ & 47 & 47 \\
\hline 18 & -18 & 13 & $\varnothing$ & 53 & 53 \\
\hline 24 & -24 & -7 & $\varnothing$ & 53 & 47 \\
\hline 36 & -36 & $\varnothing$ & $\varnothing$ & 47 & 53 \\
\hline 54 & -54 & $\varnothing$ & $\varnothing$ & 103 & 47 \\
\hline 72 & -72 & $\varnothing$ & $\varnothing$ & 47 & 47 \\
\hline 108 & -108 & $\varnothing$ & $\varnothing$ & 199 & 47 \\
\hline 162 & -162 & $\varnothing$ & $\varnothing$ & 47 & 47 \\
\hline 216 & -216 & $\varnothing$ & $\varnothing$ & 47 & 47 \\
\hline 324 & -324 & $\varnothing$ & $\varnothing$ & 47 & 103 \\
\hline 486 & -486 & $\varnothing$ & $\varnothing$ & 47 & 47 \\
\hline 648 & -648 & $\varnothing$ & $\varnothing$ & 47 & 47 \\
\hline 972 & -972 & $\varnothing$ & $\varnothing$ & 47 & 47 \\
\hline 1944 & -1944 & $\varnothing$ & $\varnothing$ & 47 & 47 \\
\hline \multicolumn{4}{|c|}{} \\
\hline \multicolumn{1}{|c|}{} & \multicolumn{3}{c}{} \\
\hline
\end{tabular}

TABLE VI. $(j=1) u_{m}= \pm 3^{s}, 0 \leq s \leq 2$

\begin{tabular}{|r|r||c|c||r|r|}
\hline \multicolumn{2}{|c||}{$u_{m}$} & \multicolumn{2}{c||}{$m$} & \multicolumn{2}{|c|}{$p$} \\
\hline 1 & -1 & $-2,-1,0,2,3,6$ & 1,9 & 103 & 47 \\
\hline 3 & -3 & $-3,5$ & 4 & 47 & 103 \\
\hline 9 & -9 & $-5,8$ & $\varnothing$ & 47 & 47 \\
\hline
\end{tabular}

\section{THE FINAL RESULT}

The results of all the previous sections are summarized as follows:

Theorem 3. The only solutions $\left(n, y_{1}, y_{2}\right)$ of the equation

$$
b_{n}= \pm 2^{y_{1}} \cdot 3^{y_{2}}, \quad n \in \mathbf{Z} \text {, }
$$

where $b_{0}=b_{1}=0, b_{2}=1, b_{n+3}=2 b_{n+2}-4 b_{n+1}+4 b_{n}$, are the 44 ones listed below.

\begin{tabular}{|c|c|c|c|c|c|c|c|c|c|c|c|}
\hline$n$ & -26 & -20 & -13 & -12 & -11 & -9 & -8 & -7 & -6 & -5 & -4 \\
\hline$b_{n}$ & $2^{-18} 3^{4}$ & $2^{-11} 3$ & $2^{-10} 3^{2}$ & $2^{-8} 3$ & $2^{-6}$ & $2^{-7} 3$ & $2^{-5}$ & $2^{-6} 3$ & $2^{-4}$ & $2^{-4}$ & $2^{-4}$ \\
\hline$n$ & -3 & -2 & -1 & 2 & 3 & 5 & 7 & 8 & 9 & 10 & 11 \\
\hline$b^{n}$ & $2^{-3}$ & $2^{-2}$ & $2^{-2}$ & 1 & 2 & $-2^{2}$ & $2^{4}$ & $2^{4}$ & $-2^{5}$ & $-2^{6}$ & $2^{6}$ \\
\hline$n$ & 12 & 14 & 15 & 16 & 17 & 18 & 19 & 20 & 22 & 24 & 25 \\
\hline$b_{n}$ & $2^{8}$ & $-2^{8} 3$ & $-2^{9}$ & $2^{11}$ & $2^{10} 3$ & $-2^{12}$ & $-2^{12} 3$ & $2^{12}$ & $2^{14}$ & $-2^{17}$ & $2^{18}$ \\
\hline$n$ & 26 & 27 & 28 & 29 & 30 & 36 & 39 & 40 & 43 & 45 & 91 \\
\hline$b_{n}$ & $2^{16} 3^{2}$ & $-2^{17} 3$ & $-2^{21}$ & $-2^{18}$ & $2^{21} 3$ & $-2^{24}$ & $2^{25} 3^{3}$ & $2^{27} 3^{2}$ & $2^{28} 3^{2}$ & $2^{29} 3^{2}$ & $2^{60} 3^{4}$ \\
\hline
\end{tabular}




\section{AN ALTERNATIVE APPROACH TO (17)}

In this section we indicate how linear forms in logarithms of algebraic numbers, in combination with a recent computational technique, can be applied to the solution of equation (17).

It is not difficult to prove the following (we omit the proof):

Lemma. If in equation (17) we have $s \geq 5$, then $m=13 \cdot 3^{\nu} \cdot M+j$, where $j \in\{0,1,4,17\}$ and $\nu \geq s-4$.

This result will be applied below. In the sequel we will assume that $s \geq 5$. We number the conjugates of $\theta$ as follows:

$$
\theta^{(1)} \simeq 0.352201129+i \cdot 1.721433237, \quad \theta^{(2)}=\overline{\theta^{(1)}}, \quad \theta^{(3)} \simeq 1.295597743 .
$$

Then $\left|\omega^{(1)}\right|=\left|\omega^{(2)}\right|>1$ and $\left|\omega^{(3)}\right|<1$. From (1) we have

$$
u_{m}=\sum_{i=1}^{3} \alpha^{(i)} \theta^{(i)} \omega^{(i)^{m}}=\sum_{i=1}^{3} \beta^{(i)} \omega^{(i)^{m}},
$$

where $\beta^{(i)}=\alpha^{(i)} \theta^{(i)}$, for $i=1,2,3$.

Let $m>17$. Then $u_{m} \neq 0$, and consequently $\left|u_{m}\right| \geq 1$. Also, $\left|\beta^{(3)} \omega^{(3)^{m}}\right|<$ $5.8 \cdot 10^{-6}$, and therefore

$$
\begin{aligned}
1 \leq\left|u_{m}\right| & \leq\left|\beta^{(1)} \omega^{(1)^{m}}+\beta^{(2)} \omega^{(2)^{m}}\right|+\left|\beta^{(3)} \omega^{(3)^{m}}\right| \\
& <\left|\beta^{(1)} \omega^{(1)^{m}}+\beta^{(2)} \omega^{(2)^{m}}\right|+5.8 \cdot 10^{-6}
\end{aligned}
$$

Then,

$$
\begin{aligned}
\left|u_{m}\right| & >0.999994 \cdot\left|\beta^{(1)} \omega^{(1)^{m}}+\beta^{(2)} \omega^{(2)^{m}}\right| \\
& =0.999994 \cdot\left|\beta^{(1)} \omega^{(1)^{m}}\right| \cdot\left|\left(-\frac{\beta^{(2)}}{\beta^{(1)}}\right) \cdot\left(-\frac{\omega^{(2)}}{\omega^{(1)}}\right)^{m}-1\right| \\
& >0.259988 \cdot\left|\omega^{(1)}\right|^{m}\left|\left(-\frac{\beta^{(2)}}{\beta^{(1)}}\right) \cdot\left(-\frac{\omega^{(2)}}{\omega^{(1)}}\right)^{m}-1\right| .
\end{aligned}
$$

We put

$$
\Lambda=\log \left(-\frac{\beta^{(2)}}{\beta^{(1)}}\right)\left(-\frac{\omega^{(2)}}{\omega^{(1)}}\right)^{m}=\log \left(-\frac{\beta^{(2)}}{\beta^{(1)}}\right)+m \cdot \log \left(\frac{\omega^{(2)}}{\omega^{(1)}}\right)+k \cdot \log (-1),
$$

where $\log$ denotes the principal branch of the logarithmic function and $k$ is some integer with $|k| \leq m+1$. Then

$$
\left|u_{m}\right|>0.259988 \cdot\left|\omega^{(1)}\right|^{m} \cdot\left|e^{\Lambda}-1\right| .
$$

If $\left|e^{\Lambda}-1\right|<0.5$, then

$$
\left|e^{\Lambda}-1\right|>0.98 \cdot|\Lambda|>0 .
$$

We can now apply the theory of linear forms in logarithms of algebraic numbers (see [7]and [2]) to find a lower bound for $|\Lambda|$ as follows: By Waldschmidt's theorem [7] we found

$$
|\Lambda|>\exp \left\{-7.1669 \cdot 10^{25} \cdot(\log m+3.991)\right\} .
$$


Therefore,

$$
\left|u_{m}\right|>0.259988 \cdot 1.3562^{m} \cdot \exp \left\{-7.1669 \cdot 10^{25} \cdot(\log m+3.991)\right\} .
$$

On the other hand, from (17) and the lemma, we have

$$
\nu \leq \frac{\log m-\log 13}{\log 3}<2.335 \cdot \log m \text {, }
$$

so that

$$
\left|u_{m}\right| \leq 8 \cdot 3^{2.335 \log m+4} .
$$

Combine the last inequality with (29) to see that

$$
m \leq 5.52 \cdot 10^{27} \text {, }
$$

from which we can find an upper bound for $s$. Indeed, in view of (32),

$$
3^{s} \leq\left|u_{m}\right| \leq 8 \cdot 3^{2.335 \log m+4},
$$

from which, in combination with (31), we get $s \leq 155$.

If $m \leq 0$, things are much easier: Let us put $m=-n$, where we may suppose that $n \geq 2$. Then, from the equality

$$
u_{m}=\beta^{(1)}\left(\omega^{(1)^{-1}}\right)^{m}+\beta^{(2)}\left(\omega^{(2)^{-1}}\right)^{m}+\beta^{(3)}\left(\omega^{(3)^{-1}}\right)^{m}
$$

it is easy to see that

$$
\left|u_{m}\right|>0.75\left|\beta^{(3)}\right|\left|\omega^{(3)^{-1}}\right|^{m}>0.252171 \cdot 1.839287^{m} \text {. }
$$

On the other hand, from $m-j=13 \cdot 3^{\nu} \cdot M$, on putting $M=-N, N>0$, we get the relation $m+j=13 \cdot 3^{\nu} \cdot N \geq 13 \cdot 3^{\nu}$ and $13 \cdot 3^{\nu} \leq m+17$, from which

$$
\nu \leq \frac{\log m}{\log 3}<0.91024 \cdot \log m \text {. }
$$

Then, in view of the lemma,

$$
\left|u_{m}\right| \leq 8 \cdot 3^{s} \leq 8 \cdot 3^{\nu+4} \leq 8 \cdot 3^{0.91024 \log m+4},
$$

and this relation, combined with (34), gives $m<18$. Thus, if $m<0$, we have to check only the values $-17 \leq m \leq-1$, and this is done trivially.

The case $m>0$ (in fact, we have supposed that $m>17$ ) requires much more effort; it is not a realistic task to check $u_{m}$ for all $m \leq 5.52 \cdot 10^{27}$ (cf. (33)); nor is it realistic to solve all equations $u_{m}=2^{r} \cdot 3^{s}$ for $0 \leq r \leq 3$ and $0 \leq s \leq 155$. Therefore, we need a practical method for reducing the very large upper bound for $m$. Note first that in (29) we may suppose $\left|e^{\Lambda}-1\right|<0.5$. Indeed, if this is not the case, then (32) and (29) imply

$$
8 \cdot 3^{2.335 \log m+4} \geq 0.12994 \cdot 1.356204^{m},
$$

which gives $m \leq 63$. Then, by the lemma, the only possible values for $m$ are $39,40,43$, and 56 , and none of them is a solution of (17). Thus, $\left|e^{\Lambda}-1\right|<0.5$ holds, which implies (30). This, in turn, in combination with (32) implies

$$
|\Lambda| \leq 2543.291 \cdot 13.00404^{\log m} \cdot 1.356203^{-m} \text {. }
$$

If $m \geq 331$, then

$$
|\Lambda|<2543.29 \cdot 1.356203^{-m / 2},
$$

and we have to solve the last inequality under the restrictions $|\Lambda|>0$ and $17<$ $m<5.52 \cdot 10^{27}$. At this point, we can apply the technique of B. M. M. de Weger 
(see $\S \S 7 \mathrm{~B}$ and 7C of [8]), which reduces the upper bound of $m$ logarithmically. This requires, however, a considerable amount of computations. After this, only the "small" values of $m$ will remain to be checked, and this can be done easily, provided we have a computer program for doing long-integer arithmetic. Indeed, in the range $m \leq 1000$, say, we have to check (in view of the lemma) only the values which are congruent to $0,1,4,17$ modulo 39 , i.e., only about 100 values, to see which of them satisfy (17).

\section{BIBLIOGRAPHY}

1. F. Beukers, The zero-multiplicity of ternary recurrences, Compositio Math. 77 (1991), 165177.

2. J. Blass, A. M. W. Glass, D. Manski, D. Meronk, and R. Steiner, Constants for lower bounds for linear forms in the logarithms of algebraic numbers. I: The general case, Acta Arith. 55 (1990), 1-14.

3. J. H. Evertse, On sums of S-units and linear recurrences, Compositio Math. 53 (1984), 225-244.

4. M. Mignotte, A note on linear recursive sequences, J. Austral. Math. Soc. Ser. A 20 (1975), 242-244.

5. __, Détermination des répétitions d'une certaine suite récurrente linéaire, Publ. Math. Debrecen 33 (1986), 297-306.

6. M. Mignotte and N. Tzanakis, Arithmetical study of recurrence sequences, Acta Arith. $\mathbf{5 7}$ (1991), 357-364.

7. M. Waldschmidt, $A$ lower bound for linear forms in logarithms, Acta Arith. 37 (1980), 257283.

8. B. M. M. de Weger, Products of prime powers in binary recurrence sequences. II, Math. Comp. 47 (1986), 729-739.

Mathematique, Universite Louis Pasteur, Strasbourg, France

E-mail address: mignotte@math.u-strasbg.fr

Department of Mathematics, University of Crete, Iraklion, Greece

E-mail address: tzanakis@grearn.bitnet 